\title{
PREVENÇÃO DO CÂNCER DO COLO DO ÚTERO: DESAFIOS E PERSPECTIVAS PARA A VACINAÇÃO CONTRA O HPV NA REGIÃO DE SAÚDE NOROESTE DO ESTADO DO RIO DE JANEIRO
}

\author{
Ana Carolina Correia MESSIAS
}

Secretaria Municipal de Saúde. São José de Ubá, Rio de Janeiro, Brasil.

Autor para correspondência: carolina-messias@ hotmail.com

DOI: http://dx.doi.org/10.18571/acbm.172

\section{RESUMO}

O trabalho se propôs a apresentar as ações preventivas do câncer do colo do útero com ênfase na vacinação anti-HPV desde a sua implantação no Sistema Único de Saúde, em 2014, até o ano de 2016, na região de saúde Noroeste Fluminense, no estado do Rio de Janeiro. Ações de prevenção e promoção da saúde fazem-se fundamentais em se tratando deste tipo de câncer, pois é sabido que este é um dos que possui maior potencial de prevenção e cura quando diagnosticado precocemente. Para a realização desta proposta foi realizado um estudo descritivo com análise de dados secundários através do Sistema de Informação do Programa Nacional de Imunização e revisão bibliográfica nos principais sítios eletrônicos e bases de dados para estudos relacionados ao câncer do colo do útero. O tempo de uso da vacina anti-HPV pelo Sistema Único de Saúde ainda é curto para afirmar que esta não tem sido bem recebida pela população e é preciso que a campanha avance por mais tempo para que se possa analisar mais profundamente os motivos que têm feito com que a procura seja ainda insatisfatória.

Palavras-chave: Câncer do colo do útero; Prevenção; Vacina anti-HPV.

\begin{abstract}
This work proposed to present preventive actions of cervical cancer with emphasis on anti-HPV vaccination since its implantation in Unified Health System, in 2014, until 2016, in the region of health Noroeste Fluminense, state of Rio de Janeiro. Actions to prevent and promote health are fundamental in treating this type of cancer, as it is known this is one of those that has greater potential for prevention and cure when diagnosed early. For the accomplishment of this proposal, a descriptive study was carried out with analysis of secondary data through the Information System of the National Program of Immunization and bibliographical revision in the main electronic sites and databases for studies related to cervical cancer. The time of use of the HPV vaccine by the Unified Health System is still short to say that it has not been well received by the population. A longer campaign is necessary for analyzing more deeply why the demand is still unsatisfactory.
\end{abstract}

Keywords: Cervical cancer; Prevention; Anti-HPV vaccine.

\section{Introdução}

De acordo com a estimativa 2016 de incidência do câncer (CA) no Brasil, do Instituto Nacional do Câncer (INCA), 16.340 novos casos de câncer do colo do útero (CCU) foram esperados. Desconsiderando os tumores de pele não melanoma, o CCU é o terceiro mais incidente no País e também na região Sudeste, com 4.940 novos casos esperados. Apenas no estado do Rio de Janeiro, 1.490 novos casos eram esperados, o que torna o CCU um importante problema de saúde pública (INCA, 2015). 
Considerando que o principal fator de risco para o desenvolvimento do CCU é a infecção pelo papilomavírus humano (HPV) e que infecções persistentes por HPV podem culminar em transformações intraepiteliais progressivas com possibilidade de evolução para lesões intraepiteliais precursoras do CCU, a vacinação contra o HPV é de extrema importância.

A região Noroeste Fluminense é composta por 14 municípios, dentre eles: Aperibé, Bom Jesus do Itabapoana, Cambuci, Cardoso Moreira, Italva, Itaocara, Itaperuna, Laje do Muriaé, Miracema, Natividade, Porciúncula, Santo Antônio de Pádua, São José de Ubá e Varre-Sai (BRASIL, 2017).

\subsection{Câncer do colo do útero}

Caracteriza-se pela replicação desordenada do epitélio de revestimento do órgão, comprometendo o estroma e no período médio entre 5 a 6 anos pode evoluir para um quadro invasivo. Aproximadamente $90 \%$ dos casos progridem a partir da neoplasia intra-epitelial cervical (NIC), mas nem toda NIC evolui para um processo invasor (INCA, 2000). A incidência de CCU é de grande evidência na faixa etária entre 20 e 29 anos e o risco aumenta rapidamente até atingir seu pico, na faixa etária entre 50 e 60 anos, em geral (INCA, 2014).

Segundo o INCA (2015; 2017b), a transmissão da infecção pelo HPV pode ocorrer tanto por via sexual, através de microfissuras na mucosa ou na pele da região anogenital, como também através do contato com a pele da vulva e região perineal, perianal e bolsa escrotal. A prevenção primária da doença consiste em evitar o risco de contágio, portanto o uso de preservativos durante a relação sexual com penetração é necessário, apesar de proteger parcialmente do contágio pelo HPV. O preservativo feminino, nesse caso, destaca-se em relação ao masculino por cobrir também a região da vulva, desde que usado desde o início da relação sexual (INCA, 2015; INCA, 2017b).

É importante ressaltar que a infecção pelo HPV é um fator necessário, mas não suficiente, para o desenvolvimento do CCU em primeira instância. É preciso que a infecção seja persistente e causada por subtipos oncogênicos de HPV para o desenvolvimento de uma neoplasia, por isso se faz imprescindível a prevenção.

Outros fatores de risco para o CCU que se pode observar, além da infecção persistente por HPV, são aqueles que estão relacionados às baixas condições socioeconômicas, início precoce da atividade sexual e uso indiscriminado de contraceptivos orais, doenças sexualmente transmissíveis (DSTs) e multiplicidade de parceiros sexuais (INCA, 2003). Outros autores incluem ainda como fatores de risco a multiparidade, o tabagismo e a alimentação pobre em determinados nutrientes, como a vitamina C, o betacaroteno e o folato (SAMPAIO; ALMEIDA, 2009); além do déficit de conhecimento, a infecção pelo vírus da imunodeficiência humana (HIV) e terapia anti-retroviral, e estado imunológico deprimido (BRITO; GALVÃO, 2010).

Em países desenvolvidos, a sobrevida média estimada em cinco anos para os casos de CCU varia de 59\% a 69\%. Já nos países em desenvolvimento, com o diagnóstico mais tardio, a sobrevida média é de $49 \%$ após cinco anos, igualando-se à média mundial de 49\%. Estes dados tornam-se de extrema importância quando se considera que, se diagnosticado precocemente, este tipo de CA é $100 \%$ prevenível (INCA, 2017c).

Diante da importância desses fatores e comportamentos de risco, que facilitam a transmissão do HPV e, por consequência o surgimento do referido CA, foram desenvolvidas duas vacinas contra esse vírus. A Gardasil@, vacina quadrivalente, com alvo para os tipos 6, 11, 16 e 18 do HPV, e a Cervarix ${ }^{\circledR}$, a vacina bivalente, contra os tipos 16 e 18 do HPV. Em junho de 2006, a Food and Drug Administration (FDA), órgão regulador para medicamentos e alimentos nos Estados Unidos, aprovou a vacina quadrivalente e a licenciou para mais de 55 países (ROGERS; LOIS; LUESLY, 2008). No Brasil, a Agência Nacional de Vigilância Sanitária (ANVISA) atestou a segurança e eficácia das duas vacinas e as tornou disponíveis no mercado brasileiro. O MS disponibiliza pelo sistema único de saúde (SUS) a versão quadrivalente da vacina. 


\subsection{Prevenção em saúde e o câncer do colo do útero}

Segundo INCA (2002), as ações de prevenção primária para o CCU são destinadas ao controle dos fatores de risco evitáveis, por exemplo, dieta pobre em determinados nutrientes, fumo, multiplicidade de parceiros e multiparidade (transmissão e contágio por HPV), falta de informação acerca da doença, seu desenvolvimento e estratégias simples para evitá-la. Portanto, a conscientização da população sobre o CA e o estímulo às mudanças nos padrões de comportamento são essenciais para a prevenção primária (CARVALHO, TONANI, BARBOSA, 2005; FERREIRA, LANA, MALTA, 2005).

Já a prevenção secundária engloba o conjunto de ações que permitem o diagnóstico precoce da doença e o seu tratamento imediato, aumentando a possibilidade de cura e, portanto, melhorando a qualidade de vida, a sobrevida e diminuindo a mortalidade por CA. Aqui inclui-se o rastreamento por exame citopatológico (ou de Papanicolaou) (INCA, 2002).

$\mathrm{O}$ rastreamento (screening) pode ser classificado de duas formas: de base populacional, quando toda a população alvo de um país é buscada; ou oportunístico, quando se aproveita a procura por ações e serviços de saúde (demanda espontânea). O método de rastreamento realizado no Brasil é de tipo oportunístico, ou seja, é realizado quando a mulher procura espontaneamente o serviço de saúde (KUSCHNIR; FAUSTO, 2014).

A inexistência atual de um cadastro universal de base populacional consistente no Brasil impede o recrutamento de mulheres como realizado em países mais desenvolvidos, como o Reino Unido, onde em 1988, a cobertura do rastreamento do CCU era de apenas $42 \%$ e a incidência era de 14 a 16 casos novos para cada 100 mil mulheres por ano. Através de cartas-convite, a cobertura saltou para $85 \%$ em 1994 e, nesse curto período e sem alterações das recomendações assistenciais vigentes, a incidência caiu aproximadamente em $50 \%$, para 10 casos novos por 100 mil mulheres. Dessa forma, através de cartas-convite, iniciou a ocorrência da migração do rastreamento oportunístico para um rastreamento organizado, de acordo com a periodicidade recomendada para as mulheres definidas como população alvo, e que progressivamente passou a controlar as mulheres em falta com esse acompanhamento (QUINN et al., 1999).

Para o caso brasileiro atual, todavia, uma possibilidade é o cadastramento de mulheres e o controle de seu comparecimento para coleta de material para exame citopatológico por profissionais integrantes da estratégia de saúde da família (ESF), fortalecendo o papel da atenção primária como porta de entrada para o sistema e de coordenação do cuidado, como já propunha o Relatório Dawson, elaborado pelo ministério da saúde do Reino Unido em 1920.

\subsection{Vacina anti-HPV}

No Brasil, o MS licenciou em 2006 a versão quadrivalente da vacina anti-HPV, contra os subtipos 6, 11, 16 e 18 do referido vírus. Os subtipos 16 e 18 são os mais comuns e responsáveis por $70 \%$ dos casos de CA. Já a versão bivalente da vacina foi licenciada em 2007 para prevenção de lesões genitais pré-cancerosas do colo do útero, relacionadas aos subtipos 16 e 18 do HPV. A ANVISA registrou, então, que a vacina quadrivalente está indicada para mulheres de 9 a 26 anos e a bivalente a partir dos 9 anos, sem restrição de idade (MINISTÉRIO DA SAÚDE, 2014).

Em 2014, o MS por meio do Programa Nacional de Imunizações (PNI), introduziu a vacina quadrivalente no calendário de vacinação e nesse mesmo ano iniciou a campanha apenas para meninas entre 11 e 13 anos contra o vírus HPV. Em conjunto, foi lançado pelo PNI o Guia Prático sobre HPV - Perguntas e Respostas, com colaboração do INCA, buscando esclarecer as principais dúvidas sobre o tema entre profissionais de saúde e o público-alvo (MINISTÉRIO DA SAÚDE, 2014). Devido à pouca idade do público-alvo para essa campanha, torna-se imprescindível a participação de pais/responsáveis em conjunto com as equipes de saúde para a tomada de decisão (GONIK, 2006, apud NADAL \& MANZIONE, 2006). 
Quando administradas antes do início da atividade sexual as vacinas têm se mostrado mais efetivas. As campanhas de vacinação devem, portanto, ter como alvo os adolescentes e os préadolescentes, induzindo a produção de 10 vezes mais anticorpos que uma infecção natural pelo HPV (MINISTÉRIO DA SAÚDE, 2014). Vale ressaltar que as vacinas não conferem proteção para $100 \%$ dos casos de CCU, portanto não substitui o rastreamento pelo exame citopatológico (ou preventivo ou Papanicolaou) quando as mulheres alcançam a idade preconizada.

Para Derchain \& Sarian (2007), a vacina ideal deveria conter os seguintes atributos: segurança, atributo essencial por se tratar de um público-alvo jovem e ainda não exposto ao vírus; economia, o preço reduzido para produção e venda facilita a disseminação por populações com menor recurso; eficácia, de modo a precisar de uma ou poucas doses e evitar a revacinação e, se possível, outra via de administração que não a injetável, aumentando a receptividade pelo públicoalvo.

As vacinas anti-HPV são elaboradas partir de partículas virais semelhantes ao vírus ou VLP (do inglês viral-like particle), produzidas por tecnologia recombinante, provenientes da proteína L1 do capsídeo viral (parte que envolve o genoma do vírus) dos subtipos de HPV, altamente purificadas e capazes de gerar resposta imunológica. "Como as VLP não contêm ácido desoxirribonucleico (DNA) viral, não são capazes de infectar células, se reproduzirem ou causarem doenças" (BOSH \& SANJOSÉ, 2003 apud ANVISA, 2011).

De acordo com Markowitz et al. (2007) apud Borsatto et al. (2011), havendo interrupção no esquema de vacinação o reinício do esquema não é necessário nem recomendado. Se a interrupção tiver ocorrido após a administração da primeira dose, a segunda deve ser administrada assim que possível e o intervalo entre a segunda e terceira doses pode ser diminuído para três meses. Se apenas a terceira dose estiver atrasada, deve ser administrada tão breve possível.

O estudo de Novaes et al. (2012) quanto ao custo-efetividade da introdução da vacina antiHPV no SUS, utilizando o método de Processo Decisório para a Introdução de Novas Vacinas (PROVAC) da Organização Pan-Americana de Saúde (OPAS), subsidiou a tomada de decisão pelo MS. O estudo considerou dados epidemiológicos, dados assistenciais e de custos relacionados ao CCU. Ainda, de acordo com este estudo, foram utilizados:

\footnotetext{
taxa de desconto para custos e benefícios; incidência e mortalidade por câncer do colo do útero; sobrevida média com DALY após o diagnóstico, por estadiamento; acesso ao tratamento no setor público e privado; custo médio por caso de câncer tratado; cobertura populacional do exame Papanicolaou e seu impacto; acesso a diagnóstico e tratamento de lesões pré-cancerosas; custo médio dos procedimentos por mulher tratada; idade de início da vacinação; esquema proposto; eficácia e cobertura esperada; custos estimados da vacina, seringas e do programa, por menina vacinada.
}

Os resultados obtidos pelo estudo de Novaes et al. (2012) indicaram que a vacinação antiHPV nos diferentes cenários possíveis é custo-efetiva no Brasil.

A meta é vacinar $80 \%$ da população alvo, o que representava 4,16 milhões de meninas em 2007. O alcance da meta de cobertura vacinal gera o fenômeno conhecido como "imunidade coletiva ou de rebanho", ou seja, reduz a incidência da transmissão mesmo entre as pessoas não vacinadas (SMITH et al., 2007).

A seguir, a quadro 1 indica a população-alvo das campanhas desde 2014 até 2017 e suas doses. 


\begin{tabular}{|c|l|l|}
\hline Ano & \multicolumn{1}{|c|}{ População-alvo } & Doses (espaço em meses) \\
\hline $\mathbf{2 0 1 4}$ & $\begin{array}{l}\text { Meninas entre 11 e 13 anos } \\
\text { Meninas entre 9 e 13 anos (indígenas) }\end{array}$ & 3 doses (0 - 6 - 60) \\
\hline $\mathbf{2 0 1 5}$ & $\begin{array}{l}\text { Meninas entre 9 e 13 anos } \\
\text { Meninas a partir dos 9 anos (indígenas) }\end{array}$ & 3 doses $(0-6-60)$ \\
\hline $\mathbf{2 0 1 6}$ & $\begin{array}{l}\text { Meninas entre 9 e 13 anos } \\
\text { Mulheres entre 9 e 26 anos (portadoras de HIV) }\end{array}$ & $\begin{array}{l}2 \text { doses }(0-6) \\
3 \text { doses }(0-2-6)\end{array}$ \\
\hline $\mathbf{2 0 1 7}$ & $\begin{array}{l}\text { Meninas entre 9 e 14 anos } \\
\text { Meninos entre 11 a 14 anos } \\
\text { Mulheres e homens entre 9 e 26 anos (portadores de HIV, } \\
\text { transplantados e pacientes oncológicos em tratamento com } \\
\text { quimioterapia ou radioterapia) }\end{array}$ & $\begin{array}{l}\text { 2 doses }(0-6) \\
3 \text { doses }(0-2-6)\end{array}$ \\
\hline
\end{tabular}

Quadro 1: História da disponibilização da vacina anti-HPV pelo SUS no Brasil, sua populaçãoalvo e doses. Fonte: BRASIL, 2014; MINISTÉRIO DA SAÚDE, 2014; SBIM, 2016.

Segundo INCA (2017b):

A duração da eficácia foi comprovada em até 9 anos, mas ainda existem lacunas de conhecimento relacionadas à duração da imunidade em longo prazo (por quanto tempo as três doses recomendadas protegem contra o contágio pelo HPV) e a necessidade de dose de reforço (aplicação de novas doses da vacina no futuro na população já vacinada).

A referida vacina é, até o momento, contra-indicada para mulheres grávidas, indivíduos acometidos por doenças agudas e com hipersensibilidade aos componentes da fórmula. Até o momento também não foi demonstrada teratogenicidade (MINISTÉRIO DA SAÚDE, 2014). No entanto, pode ser administrada em lactantes, pois as informações disponíveis não demonstram nenhum efeito prejudicial.

A administração deve ser via intramuscular $(0,5 \mathrm{~mL})$ e os eventos adversos mais observados incluem dor, inchaço e vermelhidão no local da injeção e dor de cabeça de intensidade leve a moderada (INCA, 2017d).

\subsection{Prevenção e educação em saúde}

O processo comunicativo necessita ser contínuo e direcionado aos diferentes públicos, como adolescentes; pais e responsáveis; profissionais da saúde; professores e população em geral, buscando sensibilizar e informar quanto ao tema. Podem ser utilizados diversos meios como campanhas de massa, distribuição de materiais informativos (como folderes e cartilhas), fixação de cartazes pelas unidades de saúde, estímulo a rodas de conversa nas unidades de saúde, escolas, Centro de Referência de Assistência Social (CRAS), Centro de Referência Especializado de Assistência Social (CREAS) e demais atores que se fizerem pertinentes. Mídias sociais e tecnologias como aplicativos para telefone celular também podem ser usados (MINISTÉRIO DA SAÚDE 2014; CVE, 2014).

De acordo com o informe técnico produzido pela CVE (2014), as orientações quanto à prevenção do HPV e de outras DSTs devem abranger:

- Informações em linguagem clara, consistente e culturalmente adequada, ou seja, não excessivamente técnica, de fácil compreensão pelos jovens;

- Atualização do conhecimento técnico dos profissionais envolvidos, como da área da saúde, educação e assistência social;

- Orientações quanto ao comportamento sexual e ao uso de preservativos, sejam masculinos ou femininos, e em todas as relações sexuais; 
- Informações sobre o controle do tabagismo como forma de minimizar o risco de CCU.

\subsection{Redes de atenção à saúde: o papel da atenção primária à saúde na prevenção}

O objetivo primário das RAS é prestar atenção integral, com qualidade e resolução, que atenda às reais necessidades da população, tendo em vista a atual situação epidemiológica e demográfica do País, que vem se transformando de forma acelerada e com predominância das condições crônicas (KUSCHNIR; FAUSTO, 2014).

Para que as RAS cumpram seu papel, é fundamental que a APS esteja organizada para coordenar o cuidado e ser responsável pelo fluxo do usuário nas RAS. Como estratégia, a APS deve recombinar e reordenar todos os recursos do sistema para satisfazer às necessidades, às demandas e às representações da população. Por isso, há quem sugira que a APS deve "ocupar o banco do motorista" para conduzir o sistema de atenção à saúde (SALTMAN et al., 2006).

Um grande desafio para a prevenção das condições de saúde é a mudança comportamental para estilos de vida saudáveis, e depende de esforços das pessoas e do apoio por parte dos profissionais de saúde. Para aumentar a efetividade destes processos de mudança, em especial, deve-se considerar o contexto cultural, a motivação das pessoas para mudarem e o incremento da autonomia. Apesar de ser uma decisão pessoal, a mudança de comportamento pode ser apoiada por uma equipe de saúde bem preparada na APS.

Jamouolle \& Gusso (2012) descreveram que os cuidados preventivos na APS não se limitam à prevenção primária, secundária e terciária, segundo o modelo da história natural das doenças. Um novo conceito incorporado à APS é o da prevenção quaternária que volta-se à proteção das pessoas usuárias em relação ao excesso de intervenções de rastreamento de doenças. Nisto incluem-se a medicalização dos fatores de risco, de eventos vitais e adoecimentos autolimitados; a solicitação de exames em demasia; o excesso de diagnósticos; e os pedidos de exames e tratamentos solicitados pelas pessoas usuárias.

Possivelmente ocorra na APS praticada no SUS o que se constatou em outrospaíses: a suboferta de atendimentos preventivos. Nos Estados Unidos, uma pesquisaexaminou o tempo médio gasto pelo médico de família e verificou-se um dispêndio de 3 horas por dia quando seriam necessárias 10,6 horas por dia para esses procedimentos (YARNALL et al., 2003). Uma solução possível estaria em delegar as ações preventivas a outros profissionais não médicos, conforme estabelecem as RAS.

\section{Material e Métodos}

De acordo com os dados obtidos pelo Sistema de Informação do Programa Nacional de Imunização (SIPNI) pode-se apresentar a cobertura vacinal na região supracitada, compará-la ao estado do Rio de Janeiro e elencar a situação de cada município dentro da região, criando hipóteses sobre possíveis nós nessa rede de atenção. Assim pode-se formular o seguinte objetivo geral: apresentar a cobertura da vacina anti-HPV alcançada pela região Noroeste Fluminense entre os anos de 2014 e 2016.

Para a realização deste estudo descritivo foram utilizadas análises de dados públicos fornecidos pelo SIPNI e revisão bibliográfica nos principais sítios eletrônicos e bases de dados para estudos relacionados ao CCU, como INCA, Departamento de Informática do SUS (DATASUS), Fiocruz, Biblioteca Virtual em Saúde e Scielo.

\section{Resultados}

O estado do Rio de Janeiro possui 92 municípios e em sua trajetória de regionalização na saúde foram instituídas 9 regiões de saúde, sendo: Metropolitana I, Baia da Ilha Grande, 
Metropolitana II, Baixada Litorânea, Centro Sul, Médio Paraíba, Serrana, Norte e Noroeste (SESRJ, 2017a). A criação de regiões de saúde permite que o acesso às unidades e a continuidade do cuidado em saúde sejam oferecidos em um espaço territorial que possibilite o menor deslocamento possível dos usuários do SUS.

A região de saúde Noroeste Fluminense do estado do Rio de Janeiro é composta por 14 municípios, contando com uma população de 330.093 habitantes, segundo Censo 2010 do IBGE. Dentre os municípios com maior população nessa região pode-se citar Itaperuna (95.841 habitantes), Santo Antônio de Pádua (40.589 habitantes) e Bom Jesus do Itabapoana (35.411 habitantes). Já os municípios com menor população são Laje do Muriaé (7.487 habitantes) e São José de Ubá (7.003 habitantes).

De acordo com uma nota publicada no sítio eletrônico da SES-RJ, dados do MS mostram que em 2016, apenas 37,8\% das doses da vacina anti-HPV distribuídas aos estados foram aplicadas. No Rio de Janeiro, o índice é sutilmente maior que a média nacional: $38,11 \%$. Diante disso, o estado do Rio de Janeiro orientou que os municípios reforçassem a vacinação entre os adolescentes já que a resposta imunológica conferida na faixa etária alvo da campanha é extremamente relevante, e da importância da administração antes do início da atividade sexual por garantir maior efetividade (SES-RJ, 2017c).

Ainda na nota acima referida, a SES-RJ informa através de entrevista com o secretário de estado de saúde, Luiz Antonio Teixeira Jr., que:

O Brasil foi o primeiro país da América Latina a incluir a vacina de meninos contra o HPV em seu calendário de imunização. Apesar deste fato pioneiro, ainda se observa muita resistência ao assunto e isso influencia na baixa procura dos pais para a vacinação de seus filhos. É preciso que a nossa sociedade compreenda a importância da antecipação: a faixa etária indicada para que a proteção seja mais eficiente é entre 9 e 13 anos, quando se verifica o pico de anticorpos entre meninos e meninas.

De 2014 até junho de 2017, na faixa etária entre 9 e 15 anos, foram imunizadas com a primeira dose (D1) 10,7 milhões de meninas, correspondente a 74,7\% do total de brasileiras nessa faixa etária. $47 \%$ do público-alvo (7,1 milhões de meninas) receberam o esquema vacinal completo, de duas doses, como recomenda o ministério (EBC, 2017).

Já com relação aos meninos, de janeiro a junho de 2017, 853.920 mil jovens de 12 a 13 anos foram vacinados com a D1 da vacina anti-HPV, correspondendo a 23,6\% dos 3,61 milhões de meninos nessa faixa etária que devem se imunizar (EBC, 2017).

Assim, após obtenção de dados pelo SIPNI/TABNET/DATASUS pode-se apresentara situação da região Noroeste Fluminense no contexto da prevenção do CCU, relacionado à vacinação anti-HPV e sua cobertura no período entre 2014 e 2016. De acordo com o DATASUS, os dados para o ano de 2017 são preliminares e passíveis de alteração, por isso foram desconsiderados nessa pesquisa.

Considerando o ano de 2014 o da implantação da vacina anti-HPV pelo SUS no Brasil, os resultados foram satisfatórios. Observa-se na Tabela 1, que os municípios da região de saúde Noroeste Fluminense, incluindo D1 e D2, ficaram próximos da meta de $80 \%$ de cobertura estipulada pelo MS. A referida região de saúde alcançou 97,96\% de cobertura na primeira dose e $63,92 \%$ na segunda dose. Em todas as idades nota-se considerável diferença nas coberturas entre D1 e D2, sendo D1 mais alta na maioria dos casos, provavelmente em função do direcionamento do MS em aplicar D1 nas escolas. Já D2 pode ser administrada em unidades de saúde ou de acordo com planejamento municipal.

Nota-se também a fragilidade do sistema de saúde e de informação, visto que dos 14 municípios que integram a região Noroeste Fluminense, 5 (na primeira dose) e 1 (na segunda dose) apresentaram cobertura acima de $100 \%$. Também excederam os $100 \%$ na D1 as coberturas do estado do Rio de Janeiro, região Sudeste e Brasil. Já para D2, nas mesmas localidades, as 
coberturas variam de 58,6 no estado do Rio de Janeiro a 71,37\% na região Sudeste. Aqui, denotase a importância de uma rede de atenção à saúde integrada e funcional, pois meninas de fora dos territórios adscritos foram imunizadas.

Tabela 1: Cobertura da vacina anti-HPV em meninas. D1 e D2. 2014.

\begin{tabular}{|c|c|c|c|c|c|c|c|c|c|}
\hline \multirow[t]{2}{*}{ Local } & \multicolumn{2}{|c|}{11 Anos } & \multicolumn{2}{|c|}{12 Anos } & \multicolumn{2}{|c|}{13 Anos } & \multicolumn{2}{|c|}{14 Anos $^{1}$} & Total \\
\hline & D1 & D2 & D1 & D2 & D1 & D2 & D2 & D1 & D2 \\
\hline Aperibé & 64,29 & 47,62 & 59,3 & 53,49 & 97,14 & 62,86 & 70 & 72,08 & 57,98 \\
\hline Bom Jesus do Itabapoana & 33,88 & 62,99 & 63,84 & 58,49 & 137,5 & 159,09 & 0 & 75,37 & 78,9 \\
\hline Cambuci & 69 & 12 & 82,83 & 7,07 & 80,72 & 9,64 & 0 & 77,3 & 7,45 \\
\hline Cardoso Moreira & 103,13 & 100 & 68,32 & 77,23 & 92,86 & 101,19 & 0 & 87,54 & 74,56 \\
\hline Italva & 161 & 82 & 179,21 & 63,37 & 182,56 & 83,72 & 78,85 & 173,87 & 75,43 \\
\hline Itaocara & 99,39 & 48,78 & 79,52 & 63,25 & 113,67 & 75,54 & 0 & 96,59 & 53,08 \\
\hline Itaperuna & 88,83 & 63,09 & 74,5 & 56,11 & 106,42 & 74,96 & 2,45 & 88,96 & 53,5 \\
\hline Laje do Muriaé & 56,72 & 114,71 & 84,06 & 75,36 & 105,17 & 120,69 & 0 & 80,93 & 82,56 \\
\hline Miracema & 111,45 & 94,74 & 84,48 & 109,48 & 136,79 & 175,13 & 0 & 109,36 & 107,03 \\
\hline Natividade & 79,07 & 47,69 & 87,88 & 40,15 & 102,75 & 62,39 & 25,4 & 89,19 & 45,53 \\
\hline Porciúncula & 109,62 & 69,23 & 78,88 & 67,7 & 114,18 & 94,78 & 60 & 100 & 74,61 \\
\hline Santo Antônio de Pádua & 98 & 36 & 102,63 & 50 & 163,57 & 62,4 & 39,87 & 119,26 & 49,43 \\
\hline São José de Ubá & 166 & 156 & 155,77 & 67,31 & 191,11 & 91,11 & 0 & 170,07 & 76,16 \\
\hline Varre-Sai & 131,33 & 47,62 & 78,57 & 88,1 & 110,14 & 104,35 & 0 & 106,36 & 70,34 \\
\hline Noroeste & 90,57 & 63,68 & 84,11 & 61,62 & 123,16 & 93,94 & 15,54 & 97,96 & 63,92 \\
\hline Estado do Rio de Janeiro & 103,61 & 70,2 & 92,11 & 54,32 & 118,97 & 68,93 & 38,35 & 104,08 & 58,6 \\
\hline Região Sudeste & 118,87 & 83,11 & 100,56 & 69,8 & 122,68 & 85,04 & 40,03 & 113,49 & 71,37 \\
\hline Brasil & 113,24 & 78,27 & 96,87 & 62,75 & 116,09 & 75,87 & 33,93 & 108,4 & 64,55 \\
\hline
\end{tabular}

Fonte: SIPNI/DATASUS, 2017a. ${ }^{1}$ Doses de HPV Quadrivalente administradas em meninas de 14 anos referem-se apenas àquelas que iniciaram a vacinação com D1 aos 13 anos ou vacinadas no serviço privado. Nota: 0 Dado numérico igual a 0 resultante de arredondamento de um dado originalmente positivo. D1 para primeira dose e D2 para segunda dose.

Para o ano de 2015, a tabela 2 apresenta um cenário ligeiramente pior do que o do ano anterior. Observa-se que a maioria das localidades analisadas se encontram muito abaixo da meta do MS, apresentando valores inferiores a $50 \%$ de cobertura tanto na D1 como na D2.

Na região Noroeste Fluminense, os municípios de Bom Jesus do Itabapoana, Cardoso Moreira, Italva, Porciúncula e São José de Ubá são os únicos que conseguiram atingir a meta do MS na análise por idade. A região Sudeste e o Brasil alcançaram a meta do MS apenas em meninas de 9 anos, na primeira dose.

O fato observado em 2014, de que a cobertura de D1 era consideravelmente maior que a de D2 não se repetiu em $2015 \mathrm{com}$ tanta frequência. $\mathrm{O}$ fato explica-se porque menos meninas foram imunizadas. Com isso, observou-se ligeira queda na cobertura global.

$\mathrm{Na}$ análise da cobertura total, alcançaram a meta estipulada pelo MS apenas Bom Jesus do Itabapoana e São José de Ubá, tendo este último apresentado o dado de 235,57\%. 
Tabela 2: Cobertura da vacina anti-HPV em meninas. D1 e D2. 2015.

\begin{tabular}{|c|c|c|c|c|c|c|c|c|c|}
\hline \multirow[t]{2}{*}{ Local } & \multicolumn{2}{|c|}{9 Anos } & \multicolumn{2}{|c|}{10 Anos } & \multicolumn{2}{|c|}{11 Anos } & \multirow{2}{*}{$\begin{array}{c}12 \text { Anos } \\
\text { D2 }\end{array}$} & \multicolumn{2}{|c|}{ Total } \\
\hline & D1 & D2 & D1 & D2 & D1 & D2 & & D1 & D2 \\
\hline Aperibé & 63,64 & 44,16 & 35 & 41,25 & 26,19 & 45,24 & 30,23 & 41,08 & 41,14 \\
\hline Bom Jesus do Itabapoana & 118,32 & 5,13 & 88,93 & 5,19 & 84,69 & 14,33 & 10,06 & 96,66 & 9,2 \\
\hline Cambuci & 14,71 & 1,96 & 21,78 & 3,96 & 38 & 13 & 20,2 & 24,75 & 9,29 \\
\hline Cardoso Moreira & 26,19 & 0 & 18,89 & 4,44 & 39,58 & 22,92 & 97,03 & 28,52 & 26,93 \\
\hline Italva & 93,68 & 54,74 & 93,81 & 69,07 & 45 & 99 & 39,6 & 77,05 & 71,86 \\
\hline Itaocara & 52,6 & 59,74 & 66,04 & 45,91 & 76,83 & 62,8 & 9,64 & 65,41 & 47,62 \\
\hline Itaperuna & 51,58 & 5,71 & 49,49 & 10,68 & 40,55 & 12,41 & 13,69 & 47,05 & 11,02 \\
\hline Laje do Muriaé & 44,26 & 0 & 48,44 & 0 & 29,85 & 2,99 & 5,8 & 40,63 & 2,04 \\
\hline Miracema & 69,05 & 15,24 & 61,93 & 25,23 & 25,55 & 44,05 & 43,97 & 51,6 & 33,33 \\
\hline Natividade & 65,22 & 24,35 & 59,5 & 39,67 & 35,66 & 51,16 & 28,79 & 52,88 & 39,36 \\
\hline Porciúncula & 92,86 & 47,14 & 66,67 & 33,33 & 38,46 & 44,87 & 24,84 & 65,01 & 37,93 \\
\hline Santo Antônio de Pádua & 30,69 & 4,14 & 12,84 & 8,11 & 14,67 & 12,33 & 22,37 & 19,3 & 11,31 \\
\hline São José de Ubá & 251,02 & 4,08 & 260 & 6 & 196 & 8 & 3,85 & 235,57 & 5,98 \\
\hline Varre-Sai & 42,68 & 0 & 65,85 & 0 & 83,13 & 0 & 19,05 & 63,97 & 3,23 \\
\hline Noroeste & 64,5 & 15,52 & 57,13 & 18,05 & 47,06 & 26,58 & 22,81 & 56,01 & 21,38 \\
\hline Estado do Rio de Janeiro & 72,39 & 29,57 & 61,78 & 28,34 & 47,41 & 36,73 & 38 & 60,15 & 33,1 \\
\hline Região Sudeste & 98,84 & 52,17 & 79,74 & 53,9 & 50,89 & 55,92 & 43,08 & 75,83 & 52,42 \\
\hline Brasil & 88,72 & 42,23 & 73,57 & 42,74 & 50,65 & 48,57 & 42,94 & 70,61 & 44,67 \\
\hline
\end{tabular}

Fonte: SIPNI/DATASUS, 2017b. Nota 1: Os dados constantes neste site referem-se apenas as doses aplicadas no ano de 2015 em crianças indígenas e não indígenas, convivendo ou não com HIV. Não estão sendo computadas as doses aplicadas em anos anteriores. Nota 2: 0 Dado numérico igual a 0 resultante de arredondamento de um dado originalmente positivo. Nota 3: Os dados constantes no site contabilizam para D1 meninas de 9 a 11 anos e para D2 meninas de 9 a 12 anos. D1 para primeira dose e D2 para segunda dose.

Como apresenta a tabela 3, para o ano de 2016, a vacinação anti-HPV em meninas apresentou queda acentuada em relação aos dois anos anteriores. Em algumas localidades não há dados numéricos disponíveis para algumas idades, como em Aperibé, Cardoso Moreira, Italva e Laje do Muriaé, fato que interfere na análise holística de dados, pois sua ausência no sistema de informação não significa dizer que nenhuma menina foi imunizada. Neste quesito, a fragilidade da informação em saúde e fragmentação do sistema como um todo torna-se um ponto de discussão fundamental para que a realidade deste cenário possa ser melhorada.

Pode-se considerar que a centralização da segunda dose da imunização anti-HPV nas unidades de saúde pode ser um fator complicador e sinalizar a baixa adesão das jovens (baixa cobertura). Por se tratar de uma estratégia preventiva que vai de encontro à sexualidade, em meninas jovens, considerando que uma parcela da população pode não ter recebido ainda informações suficientes sobre prevenção e criado, portanto, o entendimento de que a imunização nada tem a ver com o início precoce da atividade sexual, muitas jovens podem ser não autorizadas pelos responsáveis a irem às unidades de saúde para receber a segunda dose. Uma estratégia de imunização "extramuros" para D2, como nas escolas, poderia ser considerada uma saída interessante quando se analisam as altas coberturas na primeira dose. 
Tabela 3. Cobertura da vacina anti-HPV em meninas. D1 e D2. 2016.

\begin{tabular}{|c|c|c|c|c|c|c|c|c|c|c|}
\hline \multirow[t]{2}{*}{ Local } & \multicolumn{2}{|c|}{9 Anos } & \multicolumn{2}{|c|}{10 Anos } & \multicolumn{2}{|c|}{11 Anos } & \multicolumn{2}{|c|}{12 Anos } & \multicolumn{2}{|c|}{13 Anos } \\
\hline & D1 & D2 & D1 & D2 & D1 & D2 & D1 & D2 & D1 & D2 \\
\hline Aperibé & 24,68 & 3,9 & - & 6,25 & 1,19 & 15,48 & 1,16 & 11,63 & $\ldots$ & 4,76 \\
\hline Bom Jesus do Itabapoana & 9,16 & 1,83 & 16,61 & 8,65 & 33,88 & 9,45 & 3,77 & 24,53 & 4,73 & 6,31 \\
\hline Cambuci & 30,39 & 16,67 & 19,8 & 26,73 & 31 & 26 & 28,28 & 30,3 & 37,37 & 27,27 \\
\hline Cardoso Moreira & 13,1 & 2,38 & - & - & - & 1,04 & $\ldots$ & 0,99 & 1,98 & $\ldots$ \\
\hline Italva & 90,53 & 46,32 & 12,37 & 31,96 & 6 & 5 & 2,97 & 5,94 & $\ldots$ & $\ldots$ \\
\hline Itaocara & 94,16 & 11,04 & 34,59 & 22,01 & 36,59 & 13,41 & 13,86 & 5,42 & 2,4 & 12,57 \\
\hline Itaperuna & 21,05 & 5,41 & 7,07 & 6,2 & 3,72 & 4,69 & 3,22 & 4,7 & 2,14 & 1,2 \\
\hline Laje do Muriaé & 31,15 & 9,84 & - & 6,25 & - & 1,49 & $\ldots$ & 1,45 & 1,45 & 2,9 \\
\hline Miracema & 31,9 & 7,62 & 15,6 & 19,72 & 5,29 & 11,45 & 6,9 & 12,07 & 1,29 & 1,72 \\
\hline Natividade & 50,43 & 8,7 & 28,93 & 13,22 & 20,16 & 17,05 & 6,82 & 13,64 & 7,63 & 16,79 \\
\hline Porciúncula & 43,57 & 15 & 14,29 & 9,52 & 5,13 & 6,41 & 3,73 & 6,21 & 1,86 & 3,11 \\
\hline Santo Antônio de Pádua & 13,79 & 2,07 & 9,12 & 4,05 & 7,67 & 4,33 & 3,62 & 5,59 & 1,29 & 2,58 \\
\hline São José de Ubá & 30,61 & 4,08 & 16 & 8 & 4 & 10 & 5,77 & 9,62 & 7,41 & 5,56 \\
\hline Varre-Sai & 71,95 & 10,98 & 42,68 & 29,27 & 21,69 & 14,46 & 8,33 & 26,19 & 2,41 & 8,43 \\
\hline Noroeste Fluminense & 32,37 & 8,09 & 13,83 & 11,38 & 12,29 & 8,46 & 5,77 & 10,19 & 4,09 & 5,38 \\
\hline Estado do Rio de Janeiro & 50,34 & 17,09 & 19,47 & 18,92 & 14,05 & 16,3 & 10,04 & 13,57 & 6,4 & 8,7 \\
\hline Região Sudeste & 48,15 & 17,74 & 13,07 & 17,45 & 7,46 & 12,39 & 5,74 & 10,17 & 3,03 & 4,96 \\
\hline Brasil & 45,75 & 16,08 & 13,86 & 17,66 & 8,07 & 13,33 & 5,88 & 10,38 & 3,15 & 5,28 \\
\hline
\end{tabular}

Fonte: TABNET/DATASUS, 2017. Nota 1: ... Dado numérico não disponível. Nota 2: - Dado numérico igual a 0 não resultante de arredondamento. D1 para primeira dose e D2 para segunda dose.

\section{Discussão}

O controle do CA depende fundamentalmente de ações nas áreas da promoção da saúde, proteção específica e do diagnóstico precoce da doença. A comunicação tem papel de extrema relevância no processo de promoção da saúde, e quando ocorre de forma persuasiva tende a ser mais efetiva.

O processo de planejamento em rede inclui, em primeiro lugar, a definição de objetivos, factibilidade técnica e viabilidade política. $\mathrm{O}$ estabelecimento da imagem-objetivo ou situação ideal é, portanto, o ponto de partida. A partir disso, seguem a definição de estratégias de intervenção/cursos de ação, os responsáveis pelo desenvolvimento dessas ações e, sobretudo, os métodos avaliativos que serão utilizados para monitorar o quão próximo aos objetivos iniciais o plano está. Vale ressaltar que o planejamento em saúde é um processo flexível e, por isso, adaptável a novas situações que podem surgir em seu curso e estabelecimento de novos objetivos e metas (KUSCHNIR; FAUSTO, 2014).

Assim, podem ser identificados os possíveis nós na rede de saúde que têm possibilitado a fragmentação do sistema e impossibilitado que a meta nacional para a vacinação anti-HPV seja atingida (Quadro 2). Com isso, no processo de planejamento podem ser incluídas as operações necessárias para a mudança nesse cenário, os recursos necessários para a mudança e os resultados esperados. 


\begin{tabular}{|c|c|c|c|}
\hline Nó crítico & Operações & Recursos necessários & Resultados esperados \\
\hline $\begin{array}{l}\text { Desconhecimento da } \\
\text { equipe de saúde sobre } \\
\text { a prevenção do CCU. }\end{array}$ & $\begin{array}{l}\text { Promover educação } \\
\text { permanente à equipe } \\
\text { envolvida, visando à } \\
\text { importância das ações } \\
\text { de prevenção do CCU. }\end{array}$ & $\begin{array}{l}\text { Apostilas, Materiais de } \\
\text { mídia online para } \\
\text { consulta, Cartazes, } \\
\text { dentre outros. }\end{array}$ & $\begin{array}{l}\text { Equipe de saúde capaz } \\
\text { de orientar às } \\
\text { adolescentes e toda a } \\
\text { população sobre a } \\
\text { importância } \\
\text { prevenção de sua saúde. }\end{array}$ \\
\hline $\begin{array}{l}\text { Baixa procura de } \\
\text { adolescentes nas ESF. }\end{array}$ & $\begin{array}{l}\text { Realizar a busca ativa } \\
\text { na comunidade, e } \\
\text { orientar adolescentes e } \\
\text { pais/responsáveis a } \\
\text { importância dos } \\
\text { necessidade e } \\
\text { cuidados com a saúde. }\end{array}$ & $\begin{array}{l}\text { Busca ativa } \\
\text { comunidade. }\end{array}$ & $\begin{array}{lr}\text { Participação ativa das } \\
\text { adolescentes } & \text { nas } \\
\text { atividades de promoção } \\
\text { da saúde r e } \\
\text { acompanhamento } \\
\text { permanente. }\end{array}$ \\
\hline $\begin{array}{l}\text { Desconhecimento dos } \\
\text { usuários sobre a } \\
\text { promoção da saúde. }\end{array}$ & $\begin{array}{l}\text { Promover educação em } \\
\text { saúde para a população } \\
\text { usuária dos serviços de } \\
\text { saúde, priorizando a } \\
\text { importância da } \\
\text { prevenção do CCU. }\end{array}$ & $\begin{array}{l}\text { Consultas, visitas } \\
\text { domiciliares, } \\
\text { distribuição de } \\
\text { panfletos educativos, } \\
\text { dentre outros. }\end{array}$ & $\begin{array}{lr}\text { Despertar para } & \text { o } \\
\text { conhecimento } & \text { dos } \\
\text { usuários sobre } & \text { a } \\
\text { promoção da saúde. } & \end{array}$ \\
\hline $\begin{array}{l}\text { Dificuldades na } \\
\text { abordagem de temas } \\
\text { relacionados à saúde } \\
\text { das adolescentes antes } \\
\text { do início de atividade } \\
\text { sexual. }\end{array}$ & $\begin{array}{l}\text { Implantação de semana } \\
\text { educativa e de } \\
\text { imunização intensa } \\
\text { mensalmente na ESF } \\
\text { para atendimento em } \\
\text { massa }\end{array}$ & $\begin{array}{l}\text { Palestras educativas nas } \\
\text { escolas incluindo a } \\
\text { participação de } \\
\text { pais/responsáveis, } \\
\text { rodas de conversa nas } \\
\text { unidades de saúde e } \\
\text { assistência social } \\
\text { visando a integração } \\
\text { das famílias e da } \\
\text { comunidade sobre a } \\
\text { prevenção do CCU. }\end{array}$ & $\begin{array}{l}\text { Inclusão social e } \\
\text { englobamento das } \\
\text { adolescentes e famílias } \\
\text { nas atividades, } \\
\text { resultando em procura } \\
\text { com aumento gradual } \\
\text { do público em geral. }\end{array}$ \\
\hline
\end{tabular}

Quadro 2: Identificação de nós críticos e possíveis intervenções. Fonte: NOBRE, 2016. Modificado.

É importante ressaltar a importância de cada profissional de saúde envolvido, como agentes comunitários de saúde, enfermeiros e médicos; professores; assistentes sociais e psicólogos; e cada ator que se fizer necessário para que o maior número possível de jovens seja alcançado. A capacitação permanente dos profissionais envolvidos, a atualização sobre as mudanças que ocorrem a cada ano na faixa-etária alvo e, em 2017, a inclusão dos meninos, permite com que as equipes consigam se planejar a tempo de informar a população e reforçar a importância da prevenção do CCU antes que comece a nova etapa da campanha anti-HPV.

Como visto anteriormente, a atuação das equipes em um sistema fragmentado dificulta com que as ações em saúde sejam bem-sucedidas. Acerca disso, pode-se citar:

- Sistema de informação em saúde ineficiente: por um lado a apresentação de coberturas acima de $100 \%$ e por outro a não disponibilização de dados. A não disponibilização dos dados municipais nos sistemas nacionais de informação impede com que regiões de saúde, estados e o MS atuem de forma a auxiliar municípios em dificuldade pelo simples desconhecimento de sua real situação. Por outro lado, dados apresentando mais de $100 \%$ de cobertura também não mostram a realidade dos municípios, visto que tal fato não garante que a totalidade da sua população foi imunizada. É possível que a maior parte da população imunizada seja de regiões fronteiriças, o que levanta o questionamento sobre o funcionamento da rede da área em questão e suas dificuldades. Aqui, é importante relembrar que um sistema sem território não é universal nem pode ser classificado como rede de atenção à saúde (KUSCHNIR; FAUSTO, 2014). 
- Idade: meninas que foram imunizadas pela primeira vez entre 11 e 13 anos buscaram menos a segunda dose do que as meninas que receberam a D1 entre 9 e 10 anos. Reforçar a importância da prevenção entre as adolescentes deve ser tema central nos planejamentos em saúde, já que iniciação precoce da imunização tem demonstrado melhores resultados imunização efetiva.

- Diferenças na cobertura entre D1 e D2: em 2014, a diferença era significativa. Nos anos de 2015 e 2016 percebeu-se queda considerável na cobertura global, ou seja, menos jovens foram imunizadas de forma geral. Porém, naquelas que receberam D1 percebeu-se que procuraram mais por serviços de saúde em busca da segunda dose. O alcance de um número maior de jovens é aguardado com grande expectativa, pois a imunidade é realmente comprovada apenas após a administração das duas doses da vacina (BRASIL, 2014) conferindo efetividade da proteção nessa população.

Assim, ações podem ser formuladas pelos estados a partir da definição dos gestores correspondentes, e de acordo com a realidade dos seus municípios, visando o fortalecimento entre as equipes envolvidas e a população-alvo criando um elo entre saúde, educação e comunicação. Cada município, portanto, pode elaborar estratégias consonantes com os estados e o MS para a operacionalização dos seus serviços, como:

- Trabalho conjunto entre as secretarias de saúde, educação, criança, mulher, comunicação e sindicato das escolas privadas, por exemplo;

- Definição dos objetivos da vacinação e das responsabilidades de cada área envolvida;

- Elaboração de termos de autorização e recusa para pais e responsáveis e/ou definição de uso do termo disponibilizado pelo MS nos informes técnicos;

- Elaborar estratégias de vacinação: escolas públicas e privadas e/ou unidades de saúde; e agenda e/ou calendário escolar para vacinação;

- Promover capacitação dos profíssionais de saúde envolvidos desde as palestras sobre promoção a saúde e prevenção até os responsáveis pela vacinação;

- Criação de cartilhas para professores de biologia e ciências das escolas e material informativo para pais/responsáveis e alunos.

\section{Conclusão}

É essencial que novos objetivos e metas sejam traçados em rede, ações e serviços sejam descentralizados e desconcentrados, que todos os atores sejam inclusos neste processo (jovens, responsáveis, equipe de saúde, grupos religiosos e comunidade em geral), com reuniões planejadas e realizadas na linguagem que melhor pode ser compreendida por cada um desses atores, para que a partir disso seja criada a consciência sobre cada elo desta cadeia que culmina no surgimento de uma doença tão complexa como é o CA e, assim, o País possa alcançar lugares mais altos na prevenção do CCU.

O Brasil é um país em que, tradicionalmente, campanhas de imunização são bemsucedidas. O tempo de uso da vacina anti-HPV pelo SUS ainda é curto para afirmar que esta não tem sido bem recebida pela população e é preciso que a campanha avance por mais tempo para que se possa analisar mais profundamente os motivos que têm feito com que a procura seja ainda insatisfatória. Concomitantemente, é indispensável que o assunto continue em pauta nas reuniões estaduais e municipais, com a participação dos profissionais envolvidos e a criação de estratégias para engajar nessa campanha o maior número possível de jovens, diminuindo assim a incidência e a mortalidade por CCU. 


\section{Referências}

ANVISA. Agencia Nacional de Vigilância Sanitária. Boletim Brasileiro de Avaliação de tecnologias e Saúde. Câncer de colo de útero: A vacina para prevenção do HPV e o desafio para a melhoria da qualidade do rastreamento no Brasil. Ano VI no 17 | Dez. de 2011. Disponível em: http://portal.anvisa.gov.br/documents/33884/412285/Boletim+Brasileiro+de+Avalia\%C3\%A7\% $\mathrm{C} 3 \% \mathrm{~A} 30+\mathrm{de}+\mathrm{Tecnologias}+\mathrm{em}+\mathrm{Sa} \% \mathrm{C} 3 \% \mathrm{BAde}+\% 28 \mathrm{BRATS} \% 29+\mathrm{n} \% \mathrm{C} 2 \% \mathrm{BA}+17 / 74577 \mathrm{ea} 4-$ ed28-48fc-a8f4-7b8907f456d5. Acesso em: 18 jul. 2017.

BORSATTO, A. Z.; VIDAL, M. L. B.; ROCHA, R. C. N. P. Vacina contra o HPV e a Prevenção do Câncer do Colo do Útero: Subsídios para a Prática. Revista Brasileira de Cancerologia. 2011; 57(1): 67-74.

BRASIL. CONSELHO NACIONAL DE SECRETÁRIOS DE SAÚDE. Informe técnico sobre a vacina Papilomavírus humano (hpv) na atenção básica. 2014. Disponível em: http://portalarquivos.saude.gov.br/images/pdf/2015/junho/26/Informe-T--cnico-Introdu----ovacina-HPV-18-2-2014.pdf. Acesso em: 18. jul. 2017.

BRASIL. CONSELHO NACIONAL DE SECRETÁRIOS DE SAÚDE. Secretaria de Vigilância em Saúde. Nota Informativa $\mathbf{n}^{\mathbf{0}}$ 154, de 8 de junho de 2017. Disponível em: <https://sbim.org.br/images/files/nota-informativa-154-ms.pdf>. Acesso em: 07. jul. 2017.

BRITO, D. M. S.; GALVÃO, M. T. G. Fatores de risco para câncer de colo uterino em mulheres com HIV. Revista Rene, Fortaleza, v. 11, n. 1, p. 191-199, jan./mar. 2010.

CARVALHO, E.C., TONANI, M., BARBOSA, J.S. O uso da comunicação na prevenção e detecção precoce do câncer. In: MENDES, I. A. C., presidente. Comunicação em Enfermagem na Era Digital. $9^{\circ}$ Simpósio Brasileiro de Comunicação em Enfermagem; 2004. maio 27 - 28; Ribeirão Preto, São Paulo. p. 103.Ribeirão Preto: FIERP, 2005.

CVE. Vacina contra o papilomavírus humano (HPV) Informe técnico. São Paulo: Secretaria do Estado da Saúde: Centro de Vigilância Epidemiológica, 2014. Disponível em: <http://www.cve.saude.sp.gov.br/htm/imuni/pdf/HPV14_INFORME_TECNICO.pdf>. Acesso em: 18 jul. 2014.

DAWSON, B. Informe Dawson sobre el futuro de losservicios médicos y afines. 1920. Washington, OrganizaciónPanamericana de laSalud, Publicacion Científica nº 93, 1964.

DERCHAIN, S. F. M,; SARIAN, L. O. Z. Vacinas profiláticas para o HPV.RevBrasGinecol Obstet. 2007; 29(6):281-4.

EBC. EMPRESA BRASIL DE COMUNICAÇÃO S/A (EBC - AGÊNCIA BRASIL). Governo amplia público-alvo de vacinas de HPV para adultos até 26 anos.Publicado em: 18/08/2017. Disponível em: <http://agenciabrasil.ebc.com.br/geral/noticia/2017-08/governo-amplia-publicoalvo-de-vacinas-de-hpv-para-adultos-ate-26-anos>. Acesso em: 25 ago. 2017.

FERREIRA, Y.M., LANA, F.C.F., MALTA, D. C. Avaliação do Programa Viva Mulher no controle do câncer cérvico-uterino no estado de Minas Gerais. Rev Min. Enf. 9(2): 116-120, abr/jun, 2005. 
FREEDMAN, A.N.; SEMINARA,D.; GAIL, M.H.; HARTGE, P.; COLDITZ, G.A.; BALLARDBARBASH,R.; PFEIFFER, R.M. Cancer Risk Prediction Models: a workshop on development, evaluation and application. Journal Nat. CancerInst, 2005; May 97 (10): 715-723.

INSTITUTO NACIONAL DE CÂNCER (INCA). Estimativa 2014. Incidência de câncer no Brasil.

Disponível

em:

<http://www.inca.gov.br/bvscontrolecancer/publicacoes/Estimativa_2014.pdf>. Acesso em: 10 jul. 2017.

INSTITUTO NACIONAL DE CÂNCER (INCA). Normas e Recomendações do INCA. Prevenção e Controle de Câncer. Rev. Bras. de Cancerologia. 48(3): 317- 332. 2002.

INSTITUTO NACIONAL DE CÂNCER (INCA). Estimativa 2016. Incidência de câncer no Brasil. 2015. Disponível em: <http://www.inca.gov.br/estimativa/2016/estimativa-2016v11.pdf>. Acesso em: 07 jul. 2017.

INSTITUTO NACIONAL DE CÂNCER (INCA). Neoplasia Intra-Epitelial Cervical - NIC. Revista Brasileira de Cancerologia, São Paulo, 2000. 46(4):355-357.

INSTITUTO NACIONAL DE CÂNCER (INCA). Prevenção do câncer do colo do útero: normas e recomendações do INCA. Revista Brasileira de Cancerologia, Rio de Janeiro, v. 49, n. 4, p. 205, 2003.

INSTITUTO NACIONAL DE CÂNCER (INCA). Câncer do colo do útero. Detecção precoce. 2017c.

Disponível

em:

<http://www2.inca.gov.br/wps/wcm/connect/tiposdecancer/site/home/colo_utero/deteccao_preco ce>. Acesso em: 18 jul. 2017.

INSTITUTO NACIONAL DE CÂNCER (INCA). Câncer do colo do útero. HPV e câncer Perguntas mais frequentes. 2017d. Disponível em: <http://www2.inca.gov.br/wps/wcm/connect/tiposdecancer/site/home/colo_utero/hpv-cancerperguntas-mais-frequentes>. Acesso em: 18 jul. 2017.

INSTITUTO NACIONAL DE CÂNCER (INCA). Câncer do colo do útero. Prevenção. 2017b. Disponível em: http://www2.inca.gov.br/wps/wcm/connect/tiposdecancer/site/home/colo_utero/prevencao>. Acesso em: 18 jul. 2017.

INSTITUTO BRASILEIRO DE GEOGRAFIA E ESTATÍSTICA. IBGE. Censo 2010. Disponível em: <https://censo2010.ibge.gov.br/>. Acesso em: 20 jul. 2017.

JAMOULLE, M., GUSSO, G. Prevenção quaternária: primeiro não causar dano. In: GUSSO, G., LOPES, J. M. C. (Org.). Tratado de medicina de família e comunidade: princípios, formação e prática. Porto Alegre: Artmed, 2012.

KUSCHNIR, R.; FAUSTO, M. C. R. Gestão de Redes e Atenção à Saúde. Vol 2. Rio de Janeiro: EAD/ENSP, 2014. p. 93-106. 
MINISTÉRIO DA SAÚDE. INCA.SECRETARIA DE VIGILÂNCIA EM SAÚDE. Guia Prático Sobre HPV: Guia de Perguntas e Respostas para Profissionais de Saúde.Brasília: Ministério de Saúde, 2014.

NADAL, S. R,; MANZIONE, C. R. Vacinas Contra o Papiloma Vírus Humano. Rev brasColoproct. 2006;26(3): 337-340.

NOBRE, F. A. W. Proposta de intervenção para a profilaxia através da vacinação contra HPV na prevenção do câncer de colo do útero em uma unidade básica de saúde do município de Campo Alegre - AL. Trabalho de conclusão de curso de especialização latu sensu. 2016. Disponível em: <https://www.nescon.medicina.ufmg.br/biblioteca/imagem/proposta-intervencaoprofilaxia-atraves-vacinacao-contra-hpv.pdf>. Acesso em: 05 set. 2017.

NOVAES, H. M. D.et. al. Avaliação tecnológica de vacinas para a prevenção de infecção por papilomavírus humano (HPV): estudo de custo-efetividade da incorporação de vacina contra HPV no Programa Nacional de Imunizações/PNI do Brasil. Brasília, DF: Ministério da Saúde, 2012. Disponível em: http://portal2.saude.gov.br/rebrats/visao/estudo/ detEstudo.cfm?codigo=81\&evento=6\&v=true. Acessoem: 11 jul. 2017.

QUINN, M.et.al. Effect of screening on incidence of and mortality from cancer of cervix in England: evaluation based on routinely collected statistics. BMJ. 1999; 318;904.

ROGERS, J. L.; LOIS, L. J.; LUESLEY, D. M. Vaccines against cervical cancer. Current Opinion in Oncology, Londres. n, v. 20, n. 5, p. 570-574, Set. 2008.

SALTMAN, R. S. et al. Primary care in the driver's seat? Organizational reform in European primary care. European Observatory on Health Systems and Policies/Nuffield Trust. Maidenhead: Open University Press/McGraw Hill, 2006.

SAMPAIO, L. C.; ALMEIDA, C. F. Vitaminas antioxidantes na prevenção do câncer do colo uterino. Revista Brasileira de Cancerologia, Rio de Janeiro, v. 55, n. 3, p. 289-296, jul./ago./set. 2009.

SBIM. Nota Informativa $\mathbf{n}^{\mathbf{0}}$ 311/2016. Informa as mudanças no calendário vacinal para 2017. Disponível em: <https://sbim.org.br/images/files/nota-informativa-311.pdf>. Acesso em: 18 jul. 2017.

SECRETARIA DE SAÚDE DO ESTADO DO RIO DE JANEIRO (SES-RJ). SES orienta municípios a reforçarem vacinação de adolescentes.2017c.Disponível em: <http://www.saude.rj.gov.br/noticias/2017/06/ses-orienta-municipios-a-reforcarem-vacinacaode-adolescentes>. Acesso em: 11. Jul. 2017.

SECRETARIA DE SAÚDE DO ESTADO DO RIO DE JANEIRO (SES-RJ). Regionalização. 2017a. Disponível em: <http://www.saude.rj.gov.br/assessoria-de-regionalizacao/sobre-aregionalizacao/2017/04/regionalizacao>. Acesso em: 29 ago. 2017.

SMITH, J. S., LINDSAY, L., HOOTS, B., KEYS, J., FRANCESCHI, S., WINER, R.et. al.Human papillomavirus type distribution in invasive cervical cancer and high-grade cervical lesions: A meta-analysis update. Int. J. Cancer 2007; 121: 621-632. 
SISTEMA DE INFORMAÇÃO DO PROGRAMA NACIONAL DE IMUNIZAÇÃO - SIPNI. DATASUS. Estratégia de Vacinação contra HPV - 2014. Atualizada em: 29 ago. 2017.Disponível em: <http://pni.datasus.gov.br/consulta_hpv_14_selecao.php>. Acesso em: 29 ago. 2017a.

SISTEMA DE INFORMAÇÃO DO PROGRAMA NACIONAL DE IMUNIZAÇÃO - SIPNI. DATASUS. Estratégia de Vacinação contra HPV - 2015. Atualizada em: 29 ago. 2017.Disponível em: <http://pni.datasus.gov.br/consulta_hpv_15_selecao.php>. Acesso em: 29 ago. 2017 b.

TABNET. DATASUS. Imunizações. Dados referentes a cobertura da vacinação anti-HPV em 2016 para meninos e meninas. Atualizada em: 19 out. 2016.Disponível em: <http://tabnet.datasus.gov.br/cgi/deftohtm.exe?pni/cnv/cpnibr.def>. Acesso em: 25 ago.2017.

YARNALL, K. S. H.et al. Primary care: is there enough time for prevention?Am J Public Health, 93: 635-641, 2003. 